\title{
Tamanho da Turma e Desempenho Acadêmico dos Universitários: evidência para a UFPB
}

\author{
Ana Cláudia Annegues ${ }^{1}$ \\ Sabino Porto Júnior ${ }^{2}$ \\ Erik Figueiredo ${ }^{3}$
}

\section{Resumo}

O objetivo do presente estudo consiste em investigar a relação entre o tamanho da classe e o desempenho acadêmico de estudantes do ensino superior. Para isso, utilizaram-se dados administrativos da Universidade Federal da Paraíba (UFPB), os quais contêm informações acadêmicas e socioeconômicas, como as notas dos alunos e o background familiar. Como estratégia empírica, estimou-se um modelo de dados em painel, com a inclusão de efeitos fixos e dummies para combinações de professor-semestre e disciplina-semestre, como forma de captar a influência de fatores não observados fixos e variantes do tempo, tendo em vista a endogeneidade do tamanho da turma. Empregou-se o método de Guimarães e Portugal (2009) para as estimativas na média e regressão quantílica para dados em painel presente em Koenker (2004). O coeficiente do tamanho da turma estimado pela média se mostrou negativo, embora sua magnitude seja bastante pequena, ao passo que as estimações por regressões quantílicas mostram um efeito negativo para os alunos com pior desempenho e um efeito positivo aos estudantes com as melhores notas.

\section{Palavras-Chave}

Tamanho da Turma. Endogeneidade. Desempenho Acadêmico.

\footnotetext{
Abstract

The purpose of the present study is to investigate the relationship between class size and academic performance of higher education students. For that, administrative data from the Federal University of Paraíba (UFPB) were used, which contain information such as the students' grades and the number of students enrolled per class. As an empirical strategy, a panel data model was estimated, with the inclusion of fixed effects and dummies for combinations of

1 Pós-Doutoranda - Universidade Federal do Rio Grande do Norte

Endereço: Av. Sen. Salgado Filho, 3000 - Lagoa Nova - Natal/RN - Brasil - CEP: 59072-970.

E-mail: annegues.ana@gmail.com - ORCiD: https://orcid.org/0000-0001-6656-3824.

2 Professor - Universidade Federal do Rio Grande do Sul

Endereço: Av. João Pessoa, 52 - sala 33 - Centro - Porto Alegre/RS - Brasil - CEP: 90040-000.

E-mail: sabino@ppge.ufrgs.br - ORCiD: https://orcid.org/0000-0002-5907-3103.

3 Professor - Universidade Federal da Paraíba - Centro de Ciências Sociais Aplicadas - Campus I

Cidade Universitária - Centro - João Pessoa/PB - Brasil - CEP: 58059-900

E-mail: eafigueiredo@gmail.com - ORCiD: https://orcid.org/0000-0002-3479-3665.

Recebido: 01/12/2018. Aceite: 22/09/2019.

Editor Responsável: Marcos Yamada Nakaguma
}

(c) (i) (\$) Esta obra está licenciada com uma Licença Creative Commons Atribuição-Não Comercial 4.0 Internacional. 
teacher-semester and discipline-semester, as a way of capturing the influence of fixed and time variant unobserved factors, in view of the endogeneity of the class size. We use Guimarães and Portugal (2009) for mean estimates and the quantile regression present in Koenker (2004). The coefficient of class size estimated by the mean was negative, although its magnitude is very small, whereas estimates by quantile regression show a negative effect for students with poorer performance and a positive effect on students with better grades.

\section{Keywords}

Class Size. Endogeneity. Academic Performance.

\section{JEL Classification}

123. C51.

\section{Introdução}

A investigação dos fatores determinantes do desempenho educacional dos estudantes tem norteado grande parte das pesquisas em economia da educação. Embora a literatura não seja muito recente, não existe consenso a respeito de quais são os principais insumos da função de produção educacional e muito menos sobre os seus reais efeitos. Entender o papel desses fatores sobre o desempenho educacional é importante, na medida em que pode auxiliar as ações de políticas públicas na área da educação.

Um exemplo de variável sem papel definido sobre os resultados escolares é o tamanho das turmas. O senso comum entre estudantes, pais, professores e gestores defende que turmas menores geram melhores resultados em termos de desempenho. Os canais pelos quais esse efeito se daria são diversos. Com menos alunos o professor teria maior capacidade de identificar as necessidades e os interesses de cada estudante e de responder as suas dúvidas diretamente. Em turmas maiores os alunos tenderiam a ser mais dispersos, além de gerar problemas de disciplina que tomam um tempo expressivo da aula.

No entanto, essa visão consensual não encontra respaldo na literatura empírica. Enquanto alguns estudos encontram um efeito positivo da política de redução das turmas no desempenho escolar, outros contrapõem esses resultados mostrando um impacto pequeno ou até mesmo inexistente. Outros, ainda, destacam que mais alunos na sala pode melhorar o rendimento escolar. De acordo com Camargo (2012), a inclusão de mais um aluno, sendo 
este diferente dos demais, pode gerar um efeito spillover de conhecimento, no qual os alunos com pior desempenho podem se beneficiar da exposição a bons alunos. ${ }^{1}$

Esses resultados inconclusivos refletem em parte as dificuldades envolvidas na mensuração do efeito causal desta variável sobre os resultados educacionais. Uma delas é que o tamanho da turma não é diretamente observável, tendo de ser substituída por uma proxy. Além disso, existem razões para crer que o tamanho da turma não é definido de forma completamente exógena. De Paola et al. (2013) mostram que a equação de regressão do desempenho acadêmico contra a variável tamanho da turma possui fontes potenciais de endogeneidade, como o problema de causalidade reserva e o viés de variável omitida.

Em geral, as escolas alocam as turmas de acordo com o desempenho dos alunos. Estudantes com fraco desempenho podem ser direcionados para classes menores pelos gestores da escola, com o objetivo de melhorar as notas. Nesse sentido, há uma causalidade simultânea entre o resultado escolar e o tamanho da classe, violando, assim, o pressuposto da ortogonalidade entre o termo de erro e a variável explicativa. Em outros casos, a alocação dos estudantes em classes de diferentes tamanhos não é exógena devido à decisão dos pais, em que aqueles mais preocupados com a educação dos seus filhos podem escolher matriculá-los em escolas com turmas menores (De Paola et al. 2013). Tem-se, então, que fatores não observáveis, como a habilidade dos alunos e a preocupação dos pais, estão relacionados tanto ao rendimento do aluno quanto à formação das turmas.

Na educação superior o papel do tamanho da turma também não é bem definido e o número de evidências empíricas existentes na literatura ainda é reduzido em comparação com o número de pesquisas feitas para a educação primária e secundária. Embora o ensino universitário demande maior esforço e autonomia dos alunos, o entendimento geral entre a comunidade acadêmica é de que salas de aula lotadas atrapalham o rendimento acadêmico. Outra característica das turmas universitárias é que um mesmo professor pode ser responsável por várias turmas, ao passo que no ensino fundamental cada professor ensina uma mesma classe ao longo do período letivo.

1 Existem pesquisas que investigam o efeito da heterogeneidade na composição da turma sobre o desempenho escolar. Hidalgo-Hidalgo (2011) destaca que as diferenças quanto à idade e ao desempenho podem ser benéficas aos estudantes. Nesse sentido, turmas maiores podem representar ganhos em termos de diversidade. 
Assim como no ensino fundamental, o tamanho da turma no ensino superior também consiste em uma variável potencialmente endógena, devido à existência de fatores omitidos correlacionados tanto com o desempenho acadêmico quanto à formação das turmas. Os departamentos responsáveis por cada curso ofertam as disciplinas e determinam a capacidade máxima de alunos por turma, a qual dependerá da adequação entre o espaço físico e o número de alunos e demais características dos cursos. Os estudantes, por sua vez, escolhem as que irão cursar no semestre, dentro das matérias obrigatórias e atendendo a quantidade mínima de disciplinas optativas exigidas pela universidade. De acordo com Bettinger et al. (2014), a escolha dos estudantes não ocorre de forma aleatória, sendo definida por características relacionadas ao seu próprio rendimento acadêmico. Por exemplo, bons professores atraem mais alunos e, portanto, lecionam para turmas maiores. Em resumo, características não observadas, como a qualidade dos professores e o grau de dificuldade das disciplinas, podem impactar simultaneamente o tamanho da turma e os outcomes educacionais.

Alguns estudos propõem estratégias para contornar os problemas de endogeneidade do tamanho da turma nas escolas, como a utilização de variáveis instrumentais (Angrist e Lavy 1999) e métodos de pareamento (Camargo 2012). No ensino superior, as pesquisas existentes trabalham com dados longitudinais, o que permite o controle de características não observadas fixas no tempo, como os efeitos fixos dos alunos, dos professores e das disciplinas lecionadas (Monks e Schmidt 2011, Harding e Lamarche 2014). No entanto, se o viés surge da existência de não observáveis variantes no tempo, somente o controle pelos efeitos fixos não é suficiente para fornecer estimativas robustas do efeito do tamanho da turma. Uma solução seria encontrar uma variável instrumental que seja relacionada com o tamanho da turma, porém independente do desempenho acadêmico, o que não é uma tarefa trivial.

Outros trabalhos utilizam regras exógenas de formação de turmas como instrumentos, nos moldes da estratégia adotada por Angrist e Lavy (1999), para prever o tamanho das turmas. Nas universidades públicas brasileiras, não há uma regra exógena de formação de turmas definida; segundo o Ministério da Educação, a quantidade máxima de alunos por turma deverá ser condizente com a capacidade física das salas de aula. Assim, a abordagem de mensuração de Angrist e Lavy (1999) se torna inviável para o caso da UFPB. 
Dito isso, o objetivo do presente artigo consiste em investigar a relação entre o tamanho da turma e o rendimento acadêmico dos universitários, contornando a influência de fatores omitidos relacionados simultaneamente ao tamanho das turmas e ao desempenho acadêmico. A definição mais precisa do papel desta variável pode auxiliar as políticas públicas de educação a promover maiores ganhos à formação educacional dos estudantes. Sendo a educação considerada um bem meritório, esforços no sentido de melhorar os níveis de aprendizagem podem gerar benefícios em termos de desenvolvimento econômico num horizonte de longo prazo. ${ }^{2}$ No contexto da educação universitária, a necessidade de investigações mais aprofundadas se torna evidente, uma vez que a quantidade de alunos por turma tende a ser maior que nos demais níveis do sistema educacional (Bandiera et al. 2010, p. 01). Além disso, a alocação dos alunos entre as turmas representa custos financeiros às universidades (estrutura física, quantidade de professores) e saber a dimensão da influência do tamanho das turmas pode ajudar essas instituições a administrar de forma mais eficiente seus recursos.

Estimou-se um modelo de dados em painel com efeitos fixos relacionados aos alunos, aos professores e às disciplinas, dado que estas dimensões compõem as unidades observacionais (aluno, disciplina, professor e semestre). A inclusão de efeitos fixos visa controlar os fatores omitidos fixos no tempo que potencialmente afetam tanto o desempenho acadêmico quanto o tamanho da classe. Dada a ausência de instrumentos adequados para o tamanho da turma, a estratégia aqui adotada para controlar a parcela dos fatores omitidos que são variantes no tempo consiste na inclusão de dummies para interações entre professor e semestre e para interações entre disciplina e semestre. A utilização da dimensão de tempo fornece a variação de características não observadas dos professores e das disciplinas entre os semestres cursados, proporcionando uma aproximação das não observáveis time-varying. Tal abordagem já foi empregada em modelos empíricos no contexto de comércio internacional (ver Baltagi, Egger and Pfaffermayr 2014).

A estimação do modelo é feita via Ordinary Least Squares (OLS) para dados em painel com mais de duas dimensões. Esse método foi desenvolvido por Carneiro et al. (2008) e aperfeiçoado computacionalmente por Guimarães e Portugal (2009). Além disso, utilizou-se também a estimação

2 Bem meritório ou bem de mérito consiste em um bem (ou serviço) fornecido em benefício à sociedade. Seus ganhos não se restringem apenas à população diretamente atendida, gerando externalidades positivas à sociedade e ao país. Saúde e educação são exemplos de bens meritórios. 
via regressões quantílicas para dados em painel de Koenker (2004) a fim de verificar se o tamanho da classe possui efeitos diferenciados entre os quantis de desempenho acadêmico.

Utilizou-se uma base de dados administrativa da Universidade Federal da Paraíba (UFPB), a qual contém informações sobre os estudantes de graduação, entre os anos de 2001 e 2010.

Este banco de dados é fornecido pela Superintendência de Tecnologia da Informação (STI) da UFPB e contém as médias finais dos alunos em cada disciplina que cursou durante os semestres, os professores com os quais cursou as disciplinas, número de matriculados na turma, entre outras informações sobre a vida acadêmica dos alunos. O banco de dados também abrange variáveis socioeconômicas dos alunos obtidas junto a Comissão Permanente de Vestibular (COPERVE), como a nota no vestibular, background familiar (instrução e ocupação dos pais, renda familiar), sexo, raça e etc.

$\mathrm{O}$ artigo possui mais cinco seções, além desta introdução. $\mathrm{Na}$ seção seguinte é feita uma breve revisão da literatura sobre o efeito do tamanho da turma. Já a terceira seção destina-se a explanar os procedimentos metodológicos. A quarta seção faz uma descrição dos dados utilizados. Na quinta seção são mostrados os resultados obtidos. E por fim a sexta seção traz as conclusões gerais do estudo.

\section{Revisão de Literatura}

A literatura de educação há muito tem se interessado em investigar o real impacto do tamanho da turma sobre o aprendizado dos alunos, tendo em vista que esta variável sempre esteve no conjunto de insumos educacionais alvos de discussões e políticas sobre educação. Um dos estudos mais importantes foi desenvolvido pelo projeto Tennessee's Student Teacher Achievement Ratio (STAR), um experimento conduzido pelo estado do Tennessee, nos Estados Unidos, entre os anos de 1985 e 1989. Nesta pesquisa, 11.600 estudantes e seus professores foram alocados aleatoriamente entre turmas pequenas, com 13 até 17 estudantes, e turmas grandes, com 21 até 25 estudantes, o que permitiu mensurar o efeito do tamanho da turma através 
de uma comparação de médias. Os resultados indicaram que as crianças selecionadas para as classes menores obtiveram melhor desempenho que as selecionadas para classes maiores.

Em tese, os métodos experimentais de avaliação conseguem contornar os problemas de seleção. Porém, uma condição fundamental para que isso ocorra é a adequada implementação do experimento aleatório. As principais críticas ao STAR e às pesquisas subsequentes feitas a partir dos dados do projeto (Hoxby 2000, Leher e Ding 2011) residem em problemas de execução, como o fato de os professores saberem que são parte de um experimento. Desde então, dadas as dificuldades e os altos custos de implementação incorridos na realização de estudos de randomização, uma gama de trabalhos empíricos tem sido feita adotando estratégias de identificação alternativas. A Tabela 1 contém um sumário de algumas dessas pesquisas, descrevendo os procedimentos metodológicos e os principais resultados.

Tabela 1 - Pesquisas sobre o efeito do tamanho da turma

\begin{tabular}{|c|c|c|c|}
\hline Autores & Amostra & Metodologia & Conclusão \\
\hline Krueger (1999) & $\begin{array}{l}\text { Painel de } 11.600 \text { estudantes a partir } \\
\text { da base de dados do projeto STAR. }\end{array}$ & OLS (FE) e 2SLS & $\begin{array}{l}\text { Tamanho da turma tem impacto ne- } \\
\text { gativo sobre o rendimento escolar. }\end{array}$ \\
\hline Alam (2000) & $\begin{array}{l}\text { Dados de escolas de Bangladesh en- } \\
\text { tre Outubro de } 1994 \text { e Novembro de } \\
1995 \text { do Directorate of Primary Educa- } \\
\text { tion (DPE) }\end{array}$ & Pooled OLS & $\begin{array}{l}\text { Tamanho da turma não possui im- } \\
\text { pacto estatisticamente significativo. }\end{array}$ \\
\hline $\begin{array}{l}\text { Bressoux, } \\
\text { Kramarz e Prost } \\
(2005)\end{array}$ & $\begin{array}{l}\text { Dados de turmas da 3a série forneci- } \\
\text { dos pelo Ministério da Educação da } \\
\text { França }\end{array}$ & OLS (FE) e 2SLS & $\begin{array}{l}\text { Tamanho da classe afeta negativa- } \\
\text { mente as habilidades de leitura e } \\
\text { raciocínio matemático dos alunos }\end{array}$ \\
\hline $\begin{array}{l}\text { Blatchford, } \\
\text { Bassett e Brown } \\
(2005)\end{array}$ & $\begin{array}{l}\text { Amostra de } 257 \text { crianças entre } 10 \\
\text { anos no Reino Unido }\end{array}$ & OLS & $\begin{array}{l}\text { O tamanho da classe não afeta } 0 \\
\text { comportamento das crianças em } \\
\text { sala de aula. }\end{array}$ \\
\hline $\begin{array}{l}\text { Martins e Walker } \\
\text { (2006) }\end{array}$ & $\begin{array}{l}\text { Estudantes de graduação em econo- } \\
\text { mia entre } 2001 \text { e } 2004 \text { de uma univer- } \\
\text { sidade do Reino Unido }\end{array}$ & $\begin{array}{l}\text { Painel com efeito } \\
\text { fixo relacionado ano } \\
\text { aluno, ao professor, } \\
\text { à disciplina e ao se- } \\
\text { mestre }\end{array}$ & $\begin{array}{l}\text { Os autores não encontram nenhum } \\
\text { efeito do tamanho da turma sobre } \\
\text { desempenho acadêmico }\end{array}$ \\
\hline $\begin{array}{l}\text { Machado e } \\
\text { Vera-Hernandez } \\
\text { (2008) }\end{array}$ & $\begin{array}{l}\text { Dados administrativos da Universida- } \\
\text { de Carlos III de Madri, na Espanha, de } \\
2001 \text { a } 2006 \text {. }\end{array}$ & $\begin{array}{l}\text { Regra de formação } \\
\text { da turma pelo sobre- } \\
\text { nome como variável } \\
\text { instrumental }\end{array}$ & $\begin{array}{l}\text { Os autores não encontram nenhum } \\
\text { efeito do tamanho da turma sobre } \\
\text { desempenho acadêmico }\end{array}$ \\
\hline $\begin{array}{l}\text { De Giorgi et al. } \\
\text { (2012) }\end{array}$ & $\begin{array}{l}\text { Dados administrativos da Universida- } \\
\text { de de Bocconi na Itália. }\end{array}$ & $\begin{array}{l}\text { Número de matricu- } \\
\text { lados como } \\
\text { variável instrumental }\end{array}$ & $\begin{array}{l}O \text { aumento no tamanho da classe } \\
\text { reduz as notas }\end{array}$ \\
\hline
\end{tabular}

Fonte: Elaboração própria. 
Angrist e Lavy (1999) estimaram o impacto do tamanho da classe no rendimento escolar dos alunos em Israel. Os autores utilizaram uma regra de formação das turmas como variável instrumental para o tamanho da turma. A Maimonides' rule estabelece um número máximo de 40 alunos por sala; ultrapassado esse limite, as escolas criam uma classe adicional. Os autores encontram que turmas menores elevam as notas dos estudantes. Seguindo a mesma estratégia, Urquiola (2006) utiliza como instrumento uma regra semelhante a Maimonides' rule, na qual turmas com mais de 30 alunos passam a contar com mais de um professor, e também encontram uma relação negativa entre desempenho e tamanho da turma.

Ainda na esteira da abordagem de variáveis instrumentais, Asadullah (2005) também utiliza uma regra exógena como instrumento, desta vez para alocação dos professores. A partir de uma base de dados nacional de Bangladesh, o estudo conclui que políticas de redução de turma não são eficazes para a melhoria do rendimento escolar. Shin e Raudenbush (2011) utilizam variáveis instrumentais e um estimador de máxima verossimilhança para testar o efeito causal do tamanho da turma sobre diversas variáveis resposta que capturassem diversas habilidades das crianças, como leitura, raciocínio matemático, reconhecimento de palavras e etc. Embora os resultados mostrem que a redução do tamanho da classe melhorou o desempenho, o efeito positivo se mostrou pequeno para algumas séries.

No Brasil, Camargo (2012) testa a relação entre o desempenho escolar e o tamanho da turma avaliando o impacto de políticas de redução de turma no estado do Rio Grande do Sul. Para correção do viés de seleção, inicialmente o trabalho utiliza o Propensity Score Matching como estratégia de identificação. Este método busca eliminar o viés de seleção controlando para as diferenças em variáveis observáveis. Para contornar o viés de seleção em não observáveis, a autora utiliza outros métodos de avaliação, quais sejam o Local Average Treatment Effect (LATE) e a Regressão Descontínua (RDD). Os resultados mostraram que a política de redução de turma não teve impacto estatisticamente significativo sobre as notas dos alunos.

Utilizando os dados do Sistema de Avaliação da Educação Básica (SAEB), Oliveira (2008) aplica o Generalized Propensity Score Matching, considerando o tamanho da classe como uma variável de tratamento contínua. Além de mensurar o impacto da redução das turmas, a pesquisa também avalia os custos dessa política por meio de uma análise custo-efetividade. Embora a redução das turmas tenha elevado as notas dos alunos, ela apre- 
senta menor razão benefício-custo quando comparada a outras políticas educacionais, como o aumento da jornada escolar.

Conforme já argumentado, a discussão do efeito do tamanho da turma no âmbito dos ensinos primário e secundário constitui o foco da maior parte da literatura. Assim, são poucos os estudos que mensuram os efeitos dessa variável sobre o desempenho dos estudantes na universidade. As pesquisas existentes têm em comum a utilização de dados de alguma instituição de ensino superior em particular, os quais possibilitam o acompanhamento dos alunos ao longo da sua trajetória acadêmica na faculdade. Nesse sentido, os autores lidam com os problemas de endogeneidade através do controle dos efeitos fixos.

Kokkelenberg, Dillon e Christy (2008) testam o efeito do tamanho da classe sobre o desempenho acadêmico dos estudantes de uma universidade pública do Nordeste dos Estados Unidos, estimando um logit ordenado controlando para os efeitos fixos dos alunos e encontram uma relação negativa com as notas. Monks e Schmidt (2011) utilizam como variável dependente a nota média da turma e estimam um painel com três efeitos fixos, do professor, do curso e do semestre. Os autores também encontram um efeito negativo do aumento no tamanho da turma sobre as notas. Utilizando dados de uma universidade do Reino Unido, Bandiera, Larcinese e Rasul (2010) verificam se o tamanho da turma possui uma relação não linear com o desempenho acadêmico, testando o impacto de vários tamanhos de turma. Além disso, os autores consideram o efeito da variável ao longo da distribuição de notas e concluem que as classes maiores afetam negativamente o rendimento dos alunos, sobretudo daqueles localizados no topo da distribuição de notas.

O trabalho de Harding e Lamarche (2014) apresenta uma abordagem interessante ao considerar a interação entre as características do aluno e do professor, como a motivação do aluno e a qualidade do professor. Esse efeito interativo pode impactar a quantidade de alunos na classe na medida em que afeta a decisão do estudante de estar ou não em determinada turma. As evidências do estudo mostraram que os alunos com pior desempenho acadêmico são beneficiados por classes menores, enquanto que classes maiores beneficiam os alunos com melhor desempenho.

Tais resultados conflitantes mostram que encontrar o efeito do tamanho da turma ainda se mostra um desafio para a literatura. As diferentes es- 
tratégias de identificação disponíveis possuem limitações que tornam a investigação empírica uma tarefa difícil. O controle pelos efeitos fixos na abordagem de painel, por exemplo, não considera a existência de fatores não observados variantes no tempo. Uma solução para controlar esses fatores seria encontrar um instrumento exógeno, o que nem sempre é factível.

\section{Procedimentos Metodológicos}

\subsection{Modelo Empírico}

A relação entre o tamanho da turma e as notas dos alunos na universidade em geral pode ser formalizada em um modelo empírico semelhante ao estimado por Monks e Schmidt (2011):

$$
Y_{i s j t}=\beta_{0}+\beta_{1} \text { Classe }_{s j t}+\alpha_{i}+\delta_{j}+v_{s}+\varepsilon_{i s j t}
$$

Onde $Y_{i s j t}$ representa a média final que o aluno i obteve na disciplina s lecionada pelo professor $\mathrm{j}$ no semestre $t$. O tamanho da classe é dado pelo número de matriculados na turma. $\delta_{i}$ representa o efeito fixo relacionado aos alunos e $\alpha_{j}$ e $v_{s}$ e são os efeitos fixos do professor e da disciplina, os quais potencialmente afetam as notas dos estudantes e o tamanho da turma.

Conforme já argumentado, a introdução dos efeitos fixos procura captar a influência de características não observadas fixas no tempo. Porém, é possível que haja fatores não observáveis variantes influenciando tanto a formação da turma quanto a nota do aluno, que se não controla- dos geram estimativas viesadas dos reais efeitos das variáveis. Por exemplo, um professor com boa didática e que proporcione uma aula mais interessante contribui para atrair mais alunos para a turma que leciona, de modo que isso afeta o número de matriculados ao mesmo tempo que pode afetar o rendimento acadêmico. Para tratar esta fonte de endogeneidade seria necessário encontrar um instrumento exógeno para o tamanho da turma, o que, conforme já explanado, não é uma tarefa fácil, tendo em vista as limitações impostas pela disponibilidade de dados. 
Muitas pesquisas empregam a estratégia de Angrist e Levy (1999) utilizando uma regra de formação de turmas exógena como instrumento. No âmbito das universidades, Harding e Lamarche (2014) analisam o caso de uma universidade da Itália no qual a distribuição dos alunos entre as turmas é feita de forma aleatória através de uma loteria, de modo que o tamanho e demais características da turma definidas por este sorteio são utilizadas como instrumentos para o real número de alunos presentes na turma. No caso da UFPB, porém, a inexistência de uma regra de formação de turmas exógena aos cursos impossibilita a replicação dessas estratégias.

Além do controle pelos efeitos fixos do aluno, do professor e da disciplina, procurou-se contornar a endogeneidade do tamanho da turma através da introdução de dummies para diferentes interações entre professor e semestre cursado e interações entre disciplina e semestre cursado. Estas variáveis surgem como uma aproximação do fator não observado variante no tempo, no caso as características do professor e da disciplina. Tal estratégia será utilizada dada a ausência de instrumentos exógenos para o tamanho da turma.

Baltagi, Egger e Pfaffermayr (2014) aplicam uma abordagem de mensuração semelhante no contexto de comércio internacional. Os autores trabalham com uma estrutura de painel com efeitos em três dimensões: um efeito fixo relacionado aos pares de países com relações comerciais e interações de cada país com a dimensão de tempo do modelo, no caso o ano.

Assim, com a inclusão dos efeitos das interações professor-semestre e disciplina-semestre, o modelo descrito em (1) assume a forma abaixo:

$$
Y_{i s j t}=\beta_{0}+\beta_{1} \text { Classe }_{s j t}+\alpha_{i}+\delta_{j}+v_{s}+\delta_{j t}+v_{s t}+\varepsilon_{i s j t}
$$

Onde $\delta_{j t}$ e $v_{s t}$ representam as dummies de interação do semestre t com o professor j e a disciplina s, respectivamente.

Uma das fontes de endogeneidade do tamanho da turma está em características não observadas dos professores, as quais afetam simultaneamente o tamanho da turma e as notas dos alunos. Professores motivados e/ou com melhor didática e habilidade tendem a atrair mais alunos, aumentando o quantitativo de estudantes na classe, ao mesmo tempo estas mesmas características impactam o aprendizado dos estudantes. 


\subsection{Estimação}

O modelo de dados em painel a ser estimado possui mais de duas dimensões. Além do efeito fixo do aluno (indivíduo), tem-se os efeitos fixos de professor e disciplina e os efeitos de interações professor-semestre e disciplina-semestre. Isto demanda, portanto, um método de estimação para painéis com mais de duas dimensões. Carneiro et al. (2008) propuseram um método, o qual foi empregado na estimação de um modelo de regressão com mais de 26 variáveis, um conjunto de dados de 26 milhões de observações e dois efeitos fixos, trabalhador e firma. A sua implementação, entretanto, mostrou-se altamente custosa computacionalmente. Guimarães e Portugal (2009) desenvolveram um algoritmo que permite estimar um modelo com mais de um efeito fixo de forma mais simples.

Considere agora o seguinte modelo de regressão linear na sua forma matricial com a introdução de três efeitos fixos:

$$
Y=Z \beta+D_{1} \lambda+D_{2} \theta+D_{3} \delta+\varepsilon
$$

Em que $Z$ é uma matriz de variáveis explicativas e $D_{1}, D_{2}$ e $D_{3}$ são matrizes com alta dimensão que comportam as dummies dos efeitos fixos. Assim, têm-se as equações matriciais

$$
\left[\begin{array}{l}
\beta=\left(Z^{\prime} Z\right)^{-1} Z^{\prime}\left(Y-D_{1} \lambda-D_{2} \theta-D_{3} \delta\right) \\
\lambda=\left(D_{1}^{\prime} D_{1}\right)^{-1} D_{1}^{\prime}\left(Y-Z \beta-D_{2} \theta-D_{3} \delta\right) \\
\theta=\left(D_{2}^{\prime} D_{2}\right)^{-1} D_{2}^{\prime}\left(Y-Z \beta-D_{1} \lambda-D_{3} \delta\right) \\
\delta=\left(D_{3}^{\prime} D_{3}\right)^{-1} D_{3}^{\prime}\left(Y-Z \beta-D_{1} \lambda-D_{2} \theta\right)
\end{array}\right]
$$

as quais sugerem uma solução iterativa na qual a estimação de cada parâmetro do modelo depende dos demais. O ponto chave do procedimento de Guimarães e Portugal (2009) é que a dimensão das matrizes de .variáveis dummy não mais representa uma preocupação. Os termos $\left(D^{j} D\right)^{-1}$ são matrizes genéricas que consistem em uma transformação dos dados com as médias intragrupos. Além disso, $D_{1} \lambda, D_{2} \theta$ e $D_{3} \delta$ entram nas equações como vetores coluna contendo todos os elementos de $\lambda, \theta$ e $\delta$. O estimador usual de OLS considerando os efeitos fixos é fruto de uma regressão linear 
simples da variável dependente Y transformada (livre dos efeitos fixos) sobre o conjunto de variáveis exógenas $Z$. Aqui, em vez de transformar Y, ela é mantida como variável dependente em sua integridade e $D_{1} \lambda, D_{2} \theta$ e $D_{3} \delta$ entram na regressão como covariadas adicionais. Os coeficientes de $D_{1} \lambda$, $D_{2} \theta$ e $D_{3} \delta$ serão iguais a um e cada vetor por sua vez será formado pelos coeficientes estimados das variáveis dummies se estas fossem incluídas no modelo.

As estimativas do procedimento de Guimarães e Portugal fornecem o efeito médio do tamanho da turma. No entanto, é possível que haja uma variabilidade de efeitos dentro da distribuição das notas e a estimação na média pode subestimar (superestimar) esses impactos. Dito isso, também estimou-se o modelo descrito em (2) por regressões quantílicas, uma vez que este método fornece estimativas para diferentes pontos da distribuição de desempenho acadêmico. Outras vantagens da utilização de regressões quantílicas são: a robustez a outliers; o fornecimento de estimadores mais eficientes em comparação com os obtidos via OLS; e não necessita da hipótese de distribuição normal.

Dessa forma, estimamos os coeficientes para os quantis condicionais de desempenho acadêmico utilizando a abordagem de regressões quantílicas para dados em painel com efeitos fixos descrita por Koenker (2004). Considere o seguinte modelo

$$
Q_{y i j\left(\tau \mid x_{i j}\right)}=\alpha_{i}+x^{T} \beta(\tau)
$$

onde $\alpha_{i}$ representa a heterogeneidade não observada. O efeito das covariáveis pode depender do quantil $\tau$ de interesse, enquanto que $\alpha_{i}$ não. As estimativas dos coeficientes advêm do problema de minimização abaixo:

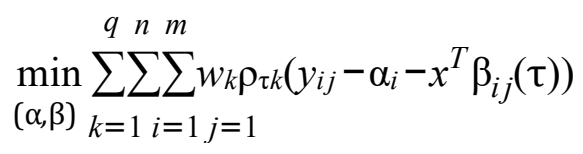

onde $\rho_{\tau k}(u)=(\tau-I(u \leq 0)) u$, como na regressão quantílica tradicional de Koenker e Basset (1978). Os pesos $w_{k}$ controlam a influência relativa dos quantis na estimação de $\alpha_{i}$.

Diferente das aplicações com OLS para dados em painel, no contexto de regressão quantílica é preciso inserir os efeitos fixos diretamente no mo- 
delo através de variáveis dummy. A utilização da abordagem de matrizes sparse auxilia na resolução desta questão, ao trabalhar apenas com os elementos da matriz diferentes de zero. ${ }^{3}$

\section{Dados}

Os dados utilizados neste estudo foram obtidos junto a Superintendência de Tecnologia da Informação (STI) da UFPB. A STI fornece uma base de dados longitudinais que contém informações acerca da vida acadêmica de todos os alunos durante a graduação: as notas finais em cada disciplina cursada, o semestre no qual ingressou e no qual finalizou o curso, o número de reprovações, além de informações sobre os professores com os quais cursou as disciplinas, como a titulação e a carga horária de trabalho.

A nota na disciplina representa a média final do aluno no período. É a média aritmética das notas obtidas durante as avaliações realizadas ao longo do semestre. Caso o aluno obtenha média maior ou igual a 7, ele é aprovado por média e esta será sua nota final na disciplina. Caso a média seja inferior a 7 e maior que 4 ele realiza um exame final; a média aritmética entre a média e a nota deste exame será a sua nota final.

Como proxy para o tamanho da turma utilizou-se o total de matriculados na disciplina cursada pelo aluno. Embora o número de matriculas não reflita necessariamente o real tamanho da turma, ele pode ser um bom previsor. A universidade não estabelece um número máximo de alunos por turma; são as coordenações dos cursos as responsáveis por alocar os alunos entre as turmas de acordo com a capacidade física das salas e a demanda por parte dos estudantes. Até a definição do número de matriculados na turma existe um período de ajuste no qual os alunos optam por seguirem matriculados ou trancarem a disciplina. Dado que o aluno pode escolher as disciplinas as quais irá cursar no semestre, é razoável supor que fatores como o interesse e a habilidade do aluno, bem como características dos professores e das disciplinas, estejam correlacionados com os tamanhos das turmas. No caso dos alunos ingressantes, embora estes sejam automatica-

A aplicação computacional da matriz sparse no contexto de regressão quantílica pode ser encontrada em Koenker e $\mathrm{Ng}(2003)$. 
mente matriculados nas disciplinas correspondentes ao primeiro semestre, eles podem trancar disciplinas e, assim, montar sua grade no semestre; a escolha de quais disciplinas trancar pode depender de características dos professores e das disciplinas, fixas ou variantes no tempo.

A Tabela 2 apresenta algumas estatísticas descritivas das variáveis. As notas nas disciplinas apresentam uma média de 7,1973, com um desvio padrão de 2,8590. As turmas da UFPB apresentam em média 34 alunos, com um desvio padrão de 15. Existem turmas que chegam a ter 200 alunos em sala.

Tabela 2 - Estatísticas Descritivas das Variáveis

\begin{tabular}{l|c|c|c|c|c}
\hline Variáveis & N.Obs. & Média & Desvio-Padrão & Mínimo & Máximo \\
\hline Nota na Disciplina & 955324 & 7,1973 & 2,8590 & 0 & 10 \\
Tamanho da Turma & 955324 & 34,2490 & 15,2405 & 1 & 206 \\
\hline
\end{tabular}

Fonte: Elaboração própria com base nos dados da STI.

A Figura 1 apresenta a distribuição do número de matriculados nas turmas para os cursos agrupados por áreas: sociais aplicadas, que incluem cursos como Economia e Administração, exatas, englobando as engenharias, Física, Matemática, Estatística e Química, as ciências humanas, com cursos como História, Filosofia e Ciências Sociais e os cursos da área da saúde.

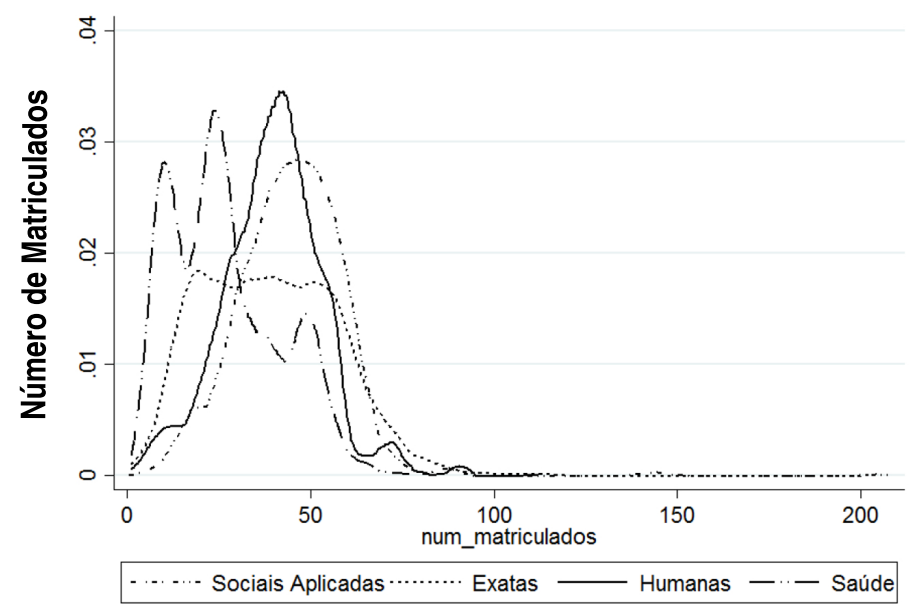

Figura 1 - Distribuição do Número de Matriculados por Turma

Fonte: Elaboração própria com base nos dados. 
Nos cursos de saúde, a distribuição se situa mais à esquerda em comparação com as demais áreas, indicando que nos cursos pertencentes a esta área tendem a ter um número menor de alunos por sala. Já para os cursos de humanas e sociais aplicadas, o número de matriculados por turma tende a ser maior. Os cursos de exatas apresentaram maior variabilidade quanto ao tamanho das turmas.

As informações de raça, gênero e background familiar foram coletadas através da aplicação de um questionário socioeconômico no momento do vestibular, organizado pela Comissão Permanente de Vestibular (COPERVE). Boa parte dos alunos não respondeu o questionário socioeconômico, ou o respondeu de forma incompleta, de modo que o banco de dados da STI com informações acadêmicas, que contém cerca 955.000 observações, passa para 131.231 observações, considerando apenas os alunos que responderam o questionário socioeconômico.

Para a estimação do efeito do tamanho da turma utilizamos a base de dados completa da STI, com todos os alunos, tendo ou não respondido o questionário socioeconômico. Mesmo que estas variáveis sejam importantes para explicar o desempenho acadêmico, são informações não variantes no tempo, cujos efeitos já são captados pelos efeitos fixos que incluímos no modelo empírico. Utilizaremos a base de dados com as informações do questionário para estimar o coeficiente do tamanho da turma para categorias de gênero e raça, como uma forma de verificar se esta variável impacta de forma heterogênea os diferentes grupos.

A Tabela 3 apresenta um perfil socioeconômico dos alunos que responderam o questionário socioeconômico da COPERVE. Aproximadamente $50 \%$ são não brancos, o que inclui negros, pardos e índios. Com relação ao gênero, a maioria dos alunos são do sexo masculino, representando $53 \%$. $23 \%$ declararam ser filhos de pais analfabetos e $13 \%$ são filhos de pais com ensino fundamental. A maior parte dos alunos tem pais com os ensinos médio e superior ou pós- graduados. 
Tabela 3 - Composição socioeconômica dos alunos da UFPB que responderam o questionário da COPERVE

\begin{tabular}{|c|c|c|c|c|c|}
\hline Variáveis & N.Obs. & Média & Desvio-Padrão & Mínimo & Máximo \\
\hline Rede onde cursou o ensino médio & 131231 & 0,4065 & 0,4911 & 0 & 1 \\
\hline Raça & 131231 & 0,5071 & 0,4974 & 0 & 1 \\
\hline Sexo & 131231 & 0,5392 & 0,4984 & 0 & 1 \\
\hline \multicolumn{6}{|c|}{ Escolaridade do Pai } \\
\hline Analfabeto & 131231 & 0,2347 & 0,4238 & 0 & 1 \\
\hline Ensino fundamental & 131231 & 0,1327 & 0,3393 & 0 & 1 \\
\hline Ensino médio & 131231 & 0,3068 & 0,4690 & 0 & 1 \\
\hline Ensino superior ou mais & 131231 & 0,3057 & 0,4607 & 0 & 1 \\
\hline
\end{tabular}

Fonte: Elaboração própria com base nos dados da STI. Nota: categorias das variáveis - sexo $(1=$ masculino; $0=$ feminino $)$, raça $(1=$ não brancos; $0=$ brancos $)$, rede do ensino médio $(1=$ escola pública; $0=$ escola privada).

\section{Resultados}

Esta seção apresenta os resultados obtidos com base na estimação pela média condicional e para os quantis da distribuição de desempenho acadêmico dos alunos. A Tabela 4 contém os coeficientes estimados via OLS considerando quatro especificações. A primeira contém apenas o tamanho da classe como variável explicativa, sem efeitos fixos e as interações entre professor- semestre e disciplina-semestre. De uma especificação a outra, esses elementos foram acrescentados como forma de gerar estimativas mais robustas e com maior grau de precisão. Destaca-se que o modelo 3 foi estimado com base na especificação do trabalho de Monks e Schmidt (2011), com efeitos fixos relacionados aos alunos, aos professores e às disciplinas. O último modelo consiste na Equação 2, em que além desses efeitos fixos incluímos as dummies de interação.

Considerando o modelo 1 , sem nenhum efeito fixo, e o modelo 2 , que inclui efeitos fixos relacionados apenas aos estudantes, o efeito do tamanho da turma seria negativo e significante, isto é, um aumento do número de matriculados na turma tende a reduzir, em média, o rendimento acadêmico dos alunos, embora a magnitude do coeficiente seja bem pequena. Com a introdução dos efeitos fixos de disciplinas e professores (modelo 3), o coeficiente do tamanho da turma não é significativo, diferente da evidência encontrada por Monks e Schmidt (2011), os quais utilizaram esta mesma especificação. 
Com as dummies de interação entre professor-semestre e disciplina-semestre, o coeficiente do tamanho da turma se torna significativo, como nos modelos 1 e 2 . De acordo com o coeficiente encontrado, uma matrícula adicional na turma tende a reduzir a nota média dos alunos na disciplina em 0,0023 pontos. Este resultado para a média segue a mesma linha das evidências encontradas por Bressoux, Kramarz e Prost (2005), para o ensino básico, e Kokkelenberg, Dillon e Christy (2008), para o ensino superior, as quais mostraram uma relação negativa desta variável com o desempenho acadêmico.

Os estudos para o ensino superior apresentam coeficientes de magnitude semelhantes aos encontrados no presente artigo. Já em Bressoux, Kramarz e Prost (2005) vê-se que a importância desta variável se mostra maior para o ensino fundamental. Tal diferença pode ser explicada, mais uma vez, pelo grau de autonomia que os alunos do ensino superior possuem, embora os efeitos de fatores externos (como o tamanho da turma) não sejam negligenciáveis.

Tabela 4 - Resultados OLS

\begin{tabular}{lcccc}
\hline Variáveis & Modelo 1 & Modelo 2 & Modelo 3 & Modelo 4 \\
\hline Tamanho da turma & $-0,0201^{* * *}$ & $-0,0006^{* \star *}$ & 0,0005 & $-0,0023^{* \star *}$ \\
Efeito Fixo (Aluno) & $(0,0002)$ & $(0,0001)$ & $(0,0002)$ & $(0,0005)$ \\
Efeitos Fixos (Disc. e Prof.) & não & sim & sim & sim \\
Dummy prof-sem & não & não & sim & sim \\
Dummy disc-sem & não & não & não & sim \\
\hline N.obs. & não & não & não & sim \\
$R^{2}$ & 955324 & 955324 & 955324 & 955324 \\
\hline
\end{tabular}

Fonte: Elaboração própria a partir das estimações.

Nota: Erro-padrão entre parênteses. ${ }^{* * *}$ p-valor $<0,01 .{ }^{* *}$ p-valor $<0,05 .{ }^{*}$ p-valor $<0,10$.

A Tabela 5 contém as estimativas por centro acadêmico da UFPB, com base na especificação mais completa (modelo 4). São eles: o Centro de Ciências Humanas, Letras e Artes (CCHLA), o Centro de Ciências Sociais Aplicadas (CCSA), o Centro de Ciências Jurídicas (CCJ), o Centro de Educação (CE), o Centro de Ciências Exatas e da Natureza, o Centro de Tecnologia (CT) (o qual reúne os cursos de engenharia) e o Centro de Ciências da Saúde (CCS).

Dos centros analisados, os coeficientes estimados para o CCHLA, o CCSA, o CT e o CSS apresentaram significância estatística; ou seja, nesses centros, um aumento no tamanho da classe tende a reduzir o desempenho 
médio dos alunos dessas áreas. Os cursos dos centros de saúde apresentam disciplinas que pela sua própria natureza demandam turmas com menos alunos, como as aulas em laboratório e as aulas de anatomia. Kokkelenberg, Dillon e Christy (2008) também investigaram a heterogeneidade do efeito do tamanho da classe entre as áreas do conhecimento e verificaram coeficientes negativos e significantes para os cursos de Matemática, Ciências Sociais, História e Computação. Já o estudo de Bettinger et al. (2014) mostrou ausência de efeito desta variável nas áreas de negócios e humanidades.

Tabela 5 - Resultados OLS por Centro Acadêmico

\begin{tabular}{|c|c|c|c|c|c|c|}
\hline \multirow[b]{2}{*}{ Variáveis } & \multicolumn{6}{|c|}{ Centros Acadêmicos } \\
\hline & CCEN & CCHLA & CE & CCSA & CT & $\operatorname{ccs}$ \\
\hline \multirow{2}{*}{ Tamanho da Turma } & $-0,0012$ & $-0,0042^{*}$ & $-0,0016$ & $-0,0024^{*}$ & $-0,0051^{\star \star \star}$ & $-0,0020^{*}$ \\
\hline & $(0,0021)$ & $(0,0022)$ & $(0,0019)$ & $(0,0013)$ & $(0,0014)$ & $(0,0011)$ \\
\hline Efeito Fixo (Aluno) & sim & $\operatorname{sim}$ & $\operatorname{sim}$ & sim & sim & $\operatorname{sim}$ \\
\hline Efeitos Fixos (Disc. e Prof.) & $\operatorname{sim}$ & sim & $\operatorname{sim}$ & $\operatorname{sim}$ & sim & $\operatorname{sim}$ \\
\hline Dummy prof-sem & $\operatorname{sim}$ & $\operatorname{sim}$ & sim & sim & $\operatorname{sim}$ & sim \\
\hline Dummy disc-sem & $\operatorname{sim}$ & $\operatorname{sim}$ & $\operatorname{sim}$ & $\operatorname{sim}$ & $\operatorname{sim}$ & sim \\
\hline N.obs. & 60422 & 94695 & 47852 & 109927 & 104783 & 153133 \\
\hline$R^{2}$ & 0,6877 & 0,6324 & 0,5623 & 0,6281 & 0,6427 & 0,6547 \\
\hline
\end{tabular}

Fonte: Elaboração própria a partir das estimações.

Nota: Erro-padrão entre parênteses. ${ }^{* * *}$ p-valor $<0,01 .{ }^{* *}$ p-valor $<0,05 .{ }^{*}$ p-valor $<0,10$.

Foi investigado também o efeito do tamanho da classe especificamente para os alunos iniciantes na faculdade, cujo período cursado coincide com o período de ingresso, e para os alunos concluintes ou egressos, nos seus semestres de conclusão (Tabela 6). Para os alunos em primeiro período, o tamanho da classe teve influência negativa e estatisticamente significante sobre o desempenho e para os alunos em conclusão de curso não foi identificado efeito do aumento no número de matriculados sobre as notas. 
Tabela 6 - Resultados OLS para alunos iniciantes e concluintes

\begin{tabular}{lcc}
\hline Variáveis & Iniciantes & Concluintes \\
\hline Tamanho da Turma & $-0,0047^{\star}$ & 0,0037 \\
Efeito Fixo (Aluno) & $(0,0017)$ & $(0,0027)$ \\
Efeitos Fixos (Disc. e Prof.) & sim & sim \\
Dummy prof-sem & sim & sim \\
Dummy disc-sem & sim & sim \\
\hline N.obs. & sim & sim \\
$R^{2}$ & 94358 & 19557 \\
\hline
\end{tabular}

Fonte: Elaboração própria a partir das estimações.

Nota: Erro-padrão entre parênteses. ${ }^{* *}$ p-valor $<0,01 .{ }^{* *}$ p-valor $<0,05 .{ }^{*}$ p-valor $<0,10$.

Os estudantes recém ingressos talvez apresentem um desempenho fortemente dependente de fatores como a estrutura da universidade. Com o passar do tempo, o aluno pode ir se adaptando ao ambiente universitário, tornando seu desempenho mais relacionado ao seu esforço individual. Dois estudos apresentam resultados divergentes quanto ao efeito do tamanho da classe no início do curso. Ngoboka e Schultz (2002) encontram um efeito negativo para alunos de cursos introdutórios de economia e Hill (1998) não encontra relação estatisticamente significante.

A Tabela 7 apresenta os coeficientes estimados do tamanho da turma para diferentes grupos de raça, gênero, escolaridade e rede escolar no ensino médio. Aqui consideramos a especificação completa, com os efeitos fixos de alunos, professores e disciplinas e as dummies de interação professor-semestre e disciplina-semestre: 
Tabela 7 - Resultado OLS por gênero, raça, rede de ensino e escolaridade dos pais

\begin{tabular}{lccc}
\hline Variáveis & Coeficiente & Erro-Padrão & $R^{2}$ \\
\hline Homens & $-0,0014$ & 0,0019 & 0,7197 \\
Mulheres & $-0,0042$ & 0,0022 & 0,7602 \\
\hline & & & \\
\hline Brancos & $-0,0022$ & 0,0019 & 0,7409 \\
Não Brancos & $-0,0036^{*}$ & 0,0020 & 0,7451 \\
\hline & Escolaridade do Pai & & \\
\hline Analfabeto & $-0,0020$ & 0,0033 & 0,7793 \\
Ensino fundamental & $-0,0139^{* *}$ & 0,0048 & 0,8070 \\
Ensino médio & $-0,0011$ & 0,0026 & 0,7643 \\
Ensino superior ou mais & 0,0027 & 0,0029 & 0,7798 \\
\hline & Rede onde cursou o ensino médio & & \\
\hline Pública & $-0,0025$ & 0,0023 & 0,7422 \\
Privada & 0,0006 & 0,0018 & 0,7460 \\
\hline
\end{tabular}

Fonte: Elaboração própria com base nas estimações.

Nota: Erro-padrão entre parênteses. ${ }^{* * *}$ p-valor $<0,01 .{ }^{* *}$ p-valor $<0,05 .{ }^{*}$ p-valor $<0,10$.

Dentre as categorias analisadas, apenas os filhos não brancos de pais com nível fundamental apresentaram coeficientes do tamanho da turma estatisticamente significantes e negativos. Ou seja, em média, um aluno adicional na turma reduz as notas desses estudantes nas disciplinas. Importante ressaltar que estas estimativas se baseiam em um conjunto de dados com um número reduzido de observações, afetando, possivelmente, a significância estatística destes coeficientes.

Além das heterogeneidades de gênero e raça, é possível que alunos com diferentes níveis de desempenho acadêmico apresentem diferentes respostas às mudanças no tamanho da turma. Nesse sentido, a regressão quantílica permite visualizar os coeficientes do tamanho da turma para diferentes quantis da variável dependente. As Tabelas 8 e 9 sintetizam os coeficientes estimados para os quantis $0,05,0,25,0,50,0,75$ e 0,95 , com a inclusão dos efeitos fixos de alunos, professores e disciplinas e das dummies de interação, respectivamente. 
Tabela 8 - Regressão Quantílica - Efeitos Fixos (FE)

\begin{tabular}{|c|c|c|c|c|c|}
\hline \multirow{2}{*}{ Variáveis } & \multicolumn{5}{|c|}{ Quantis } \\
\hline & Q_05 & Q_25 & Q_50 & Q_75 & Q_95 \\
\hline & $-0,0053^{\star * *}$ & $-0,0062^{\star \star \star}$ & $-0,0011^{* * *}$ & $0,0038^{\star \star *}$ & $0,0173^{* \star \star}$ \\
\hline Tamanho da turma & $(0,0009)$ & $(0,0001)$ & $(0,0001)$ & $(0,0001)$ & $(0,0004)$ \\
\hline Efeito Fixo (Aluno) & $\operatorname{sim}$ & $\operatorname{sim}$ & $\operatorname{sim}$ & $\operatorname{sim}$ & $\operatorname{sim}$ \\
\hline Efeitos Fixos (Disc. e Prof.) & $\operatorname{sim}$ & $\operatorname{sim}$ & $\operatorname{sim}$ & $\operatorname{sim}$ & $\operatorname{sim}$ \\
\hline N.obs. & 955324 & 955324 & 955324 & 955324 & 955324 \\
\hline
\end{tabular}

Fonte: Elaboração própria a partir das estimações.

Nota: Erro-padrão entre parênteses. ${ }^{* *}$ p-valor $<0,01 .{ }^{* *}$ p-valor $<0,05 .{ }^{*}$ p-valor $<0,10$.

Com o efeito fixo, nota-se que há variabilidade de efeitos entre os quantis e que a estimativa com relação à média tende a mascarar as respostas dos alunos com diferentes níveis de habilidade. Os alunos localizados no quantil 0,25 são os mais prejudicados em termos de rendimento acadêmico por aumentos no tamanho da turma. Já entre os estudantes mais habilidosos, o sinal do coeficiente se torna positivo e significante, indicando que para esses alunos um aluno a mais na classe aumenta a sua nota, contrariando a tese dos benefícios associados às políticas de redução de turmas.

Tabela 9 - Regressão Quantílica - Efeitos Fixos (FE) + Dummies de Interação

\begin{tabular}{|c|c|c|c|c|c|}
\hline \multirow[b]{2}{*}{ Variáveis } & \multicolumn{5}{|c|}{ Quantis } \\
\hline & Q_05 & Q_25 & Q_50 & Q_75 & Q_95 \\
\hline \multirow[t]{2}{*}{ Tamanho da turma } & $-0,0149^{\star \star *}$ & $-0,0083^{\star \star *}$ & $-0,0027^{\star \star *}$ & $0,0024^{* * *}$ & $0,0132^{* \star *}$ \\
\hline & $(0,0008)$ & $(0,0001)$ & $(0,0003)$ & $(0,0001)$ & $(0,0003)$ \\
\hline Efeito Fixo (Aluno) & $\operatorname{sim}$ & $\operatorname{sim}$ & $\operatorname{sim}$ & sim & $\operatorname{sim}$ \\
\hline Efeitos Fixos (Disc. e Prof.) & $\operatorname{sim}$ & $\operatorname{sim}$ & $\operatorname{sim}$ & $\operatorname{sim}$ & $\operatorname{sim}$ \\
\hline Dummy prof-sem & $\operatorname{sim}$ & $\operatorname{sim}$ & $\operatorname{sim}$ & $\operatorname{sim}$ & $\operatorname{sim}$ \\
\hline Dummy disc-sem & $\operatorname{sim}$ & $\operatorname{sim}$ & $\operatorname{sim}$ & $\operatorname{sim}$ & $\operatorname{sim}$ \\
\hline N.obs. & 955324 & 955324 & 955324 & 955324 & 955324 \\
\hline
\end{tabular}

Fonte: Elaboração própria a partir das estimações.

Nota: Erro-padrão entre parênteses. ${ }^{* * *}$ p-valor $<0,01 .{ }^{* *}$ p-valor $<0,05 .{ }^{*}$ p-valor $<0,10$. 
$\mathrm{Na}$ especificação mais completa, com os efeitos fixos e as dummies para as interações professor-semestre e disciplina-semestre, os sinais dos coeficientes se mantêm, embora a magnitude de alguns efeitos tenha diminuído e de outros tenha aumentado. Sendo assim, somente a presença dos efeitos fixos não é suficiente para controlar a influência de todos os fatores omitidos; o controle dos confounders variantes no tempo são importantes para minimizar possíveis sub e sobreestimações.

Os alunos com melhor desempenho aumentam suas notas em 0,0132 pontos com um aluno adicional, enquanto que os alunos com pior desempenho apresentam redução de 0,0149 pontos. Em suma, turmas maiores geram efeitos negativos sobre o desempenho dos alunos que já vem apresentando baixa performance, porém tendem a melhorar o desempenho dos estudantes com melhor rendimento.

A estratégia de identificação mais robusta utilizada por Harding e Lamarche (2014) só é possível tendo em vista que a formação das turmas na universidade analisada pelos autores permitiu a construção de instrumentos exógenos. As evidências aqui encontradas, embora com uma estratégia mais simples, caminharam no mesmo sentido das encontradas pelos autores: em salas maiores, alunos menos habilidosos têm seu desempenho acadêmico afetado de forma adversa, enquanto que os melhores estudantes melhoram seu desempenho. Segundo eles, os alunos com menores notas se beneficiam de classes menores por terem maior atenção do professor às suas necessidades educacionais. Já com relação aos melhores alunos, os autores pontuam que classes maiores proporcionam um ambiente de maior interação e competição com os demais, o que atua como um dispositivo de motivação, impulsionando o desempenho desses estudantes. Em classes maiores, estudantes mais hábeis tenderiam a monopolizar e dominar o ambiente, enquanto que os alunos menos habilidosos tenderiam a restringir sua participação em sala de aula.

\section{Considerações Finais}

O presente artigo teve por objetivo investigar como o tamanho das turmas pode afetar o desempenho acadêmico dos universitários, utilizando dados de desempenho acadêmico da Universidade Federal da Paraíba. Uma das 
dificuldades incorridas na estimação do efeito do tamanho da classe está no fato de esta variável ser endógena, devido à existência de fatores não observados correlacionados tanto com o número de alunos por sala quanto com o rendimento dos estudantes.

Dada a dificuldade de encontrar instrumentos exógenos, a estratégia empírica aqui utilizada consistiu em estimar um modelo de dados em painel com efeitos fixos relacionados ao aluno, ao professor e à disciplina e a inclusão de dummies para cada interação professor-semestre e disciplina-semestre. A inclusão de efeitos relacionados ao tempo surge como forma de capturar fatores não observados variantes no tempo.

Os resultados mostraram que, em média, há uma relação negativa entre as notas dos alunos e o tamanho da turma, ou seja, a quantidade de alunos em sala afeta negativamente o desempenho acadêmico médio na disciplina. Este resultado também é verificado nas estimativas feitas para alguns dos centros acadêmicos com cursos de engenharia, negócios, ciências exatas e saúde.

Ao estender a análise para toda a distribuição de notas e levando em conta os fatores fixos e variantes no tempo, pela aproximação com as dummies de interação com o semestre cursado, verificou-se que os alunos com pior desempenho acadêmico são os mais prejudicados com o aumento na quantidade de alunos na turma. Já para os alunos no topo da distribuição de notas, a relação se mostrou positiva, ou seja, esses estudantes têm seu desempenho acadêmico impulsionado com o aumento no tamanho da turma.

Os resultados encontrados revelam não haver consenso acerca de um modelo ideal de formação de turmas, considerando as particularidades existentes em cada aluno nas respostas a esta variável. A estratégia empírica adotada constitui uma alternativa à ausência de instrumentos e fornece resultados compatíveis com evidências da literatura que utilizaram métodos para lidar com fontes de endogeneidade. Ainda assim, o tema carece de maiores investigações empíricas, sobretudo no ensino superior, detentor de boa parte dos recursos públicos direcionados à educação. 


\section{Referências}

Angrist, J. D.; Lavy, V. "Using Maimonides' rule to estimate the effect of class size on scholastic achievement." The Quarterly Journal of Economics 114, no. 2: 533-575, 1999.

Asadullah, M. N. "The effect of class size on student achievement: evidence from Bangladesh."

Applied Economics Letters 12, no. 4: 217-221, 2005.

Baltagi, B.; Egger, P; Pfaffermayr, M. "Panel data gravity models of international trade." CESifo working papers, no. 4616, 2014.

Bandiera, O.; Larcinese, V; Rasul, I. "Heterogeneous class size effects: New evidence from a panel of university students.” The Economic Journal 120, no. 549: 1365-1398, 2010.

Bettinger, E.; Fox, L.; Loeb, S.; Taylor, E. "Changing distributions: How online college classes alter student and professor performance.” Working Paper, Stanford University, 2014.

Blatchford, P.; Bassett, P.; Brown, P. “Teachers' and Pupils' Behavior in Large and Small Classes: A Systematic Observation Study of Pupils Aged 10 and 11 Years. Journal of Educational Psychology 97, no. 3: 454, 2005.

Bressoux, P.; Kramarz, F.; Prost, Corinne. "Teachers' training, class size and students' outcomes: evidence from third grade classes in France." Document de travail CREST, décembre, 2005.

Camargo, J. "O efeito do tamanho da turma sobre o desempenho escolar: uma avaliação do impacto da "enturmação' no ensino fundamental do Rio Grande do Sul”. 2012.

Carneiro, A., Guimarães, P.; Portugal, P. "Real Wages and the Business Cycle: Accounting for Worker and Firm Heterogeneity". Unpublished manuscript, 2008.

De Giorgi, G.; Pellizzari, M.; Woolston, W. G. "Class size and class heterogeneity."

Journal of the European Economic Association 10, no. 4: 795-830, 2012.

De Paola, M.; Ponzo, M.; Scoppa, V. "Class size effects on student achievement: heterogeneity across abilities and fields." Education Economics 21, no. 2: 135-153, 2013.

Ding, W.; Lehrer, S. F. "Experimental estimates of the impacts of class size on test scores: robustness and heterogeneity.” Education Economics 19, no. 3: 229-252, 2011.

Guimarães, P.; Portugal, P. “A Simple Feasible Alternative Procedure to Estimate Models with High-Dimensional Fixed Effects”, IZA DP No 3935, 2009

Harding, M.; Lamarche, Cs. "Estimating and testing a quantile regression model with interactive effects." Journal of Econometrics 178: 101-113, 2014.

Hidalgo-Hidalgo, M. "On the optimal allocation of students when peer effects are at work: tracking vs. mixing." SERIES, 2(1): 31-52, 2011.

Hill, Mary Callahan. "Class size and student performance in introductory accounting courses: Further evidence." Issues in Accounting Education 13, no. 1: 47, 1998.

Hoxby, C. M. "The effects of class size on student achievement: New evidence from population variation." The Quarterly Journal of Economics 115, no. 4: 1239-1285, 2000.

Koenker, R.; Bassett Jr., Gilbert. "Regression quantiles." Econometrica: Journal of the Econometric Society (1978): 33-50.

Koenker, R.; NG, P. “SparseM: A sparse matrix package for R*.”Journal of Statistical Software 8, no.6: 1-9, 2003.

Koenker, R. "Quantile regression for longitudinal data." Journal of Multivariate Analysis 91, no.1: 74-89, 2004.

Kokkelenberg, E. C.; Dillon, Michael; Christy, Sean M. "The effects of class size on student grades at a public university." Economics of Education Review 27, no. 2: 221-233, 2008.

Krueger, A. B. "Economic considerations and class size." The Economic Journal 113, no. 485, 2003. 
Machado, M. Pinto; Vera Hernandez, M. "Does class-size affect the academic performance of first year college students." Retrieved March 30: 2011, 2008.

Martins, P.; Walker, I. "Student achievement and education production: a case study of the effect of class attendance." mimeo, 2006.

Monks, J.; Schmidt, Robert M. "The impact of class size on outcomes in higher education." The BE Journal of Economic Analysis \& Policy 11, no. 1, 2011.

Oliveira, J. M. de. "Custo-efetividade de políticas de redução do tamanho da classe e ampliação da jornada escolar: uma aplicação de estimadores de matching." Rio de Janeiro: Banco Nacional de Desenvolvimento Econômico e Social, 2010.

Shin, Y;; Raudenbush, S. W. "The causal effect of class size on academic achievement: Multivariate instrumental variable estimators with data missing at random." Journal of Educational and Behavioral Statistics 36, no. 2: 154-185, 2011.

Urquiola, M. "Identifying class size effects in developing countries: Evidence from rural Bolivia." The Review of Economics and Statistics 88, no. 1: 171-177, 2006. 